\title{
Cirugía robótica en el Centro Médico ABC: experiencia en los primeros 500 procedimientos realizados
}

Carlos Vásquez-Lastra ${ }^{*}$, Cesar Decanini-Terán², Antonio Maffuz-Aziz³, Jaime Alfaro-Alfaro4, José A. Huante-Pérez ${ }^{1}$, Enrique Wolpert-Barraza ${ }^{5}$, Luis F. Sánchez-Marle ${ }^{5}$ y Arturo Gutiérrez-Hernández ${ }^{6}$ ${ }^{1}$ Departamento de Urología; ${ }^{2}$ Departamento de Cirugía; ${ }^{3}$ Departamento de Cirugía oncológica; ${ }^{4}$ Departamento de Ginecología; ${ }^{5}$ Dirección Médica; ${ }^{6}$ Gerencia de la Línea de servicio de Cirugía. Comité de Cirugía Robótica, Centro Médico ABC. Ciudad de México, México

\section{Resumen}

Antecedentes: La cirugía robótica se utiliza en múltiples especialidades quirúrgicas a nivel mundial. Objetivo: Documentar la experiencia inicial del programa de cirugía robótica en un hospital de práctica privada. Material y método: Se incluyen las primeras 500 cirugías robóticas realizadas en el Centro Médico ABC, abarcando un periodo de tres años. Se documentan especialidades involucradas así como datos transoperatorios principales. Resultados: De 500 pacientes, 367 (73.4\%) fueron de sexo masculino y 133 (26.4\%) de sexo femenino. Las tres cirugías más realizada fueron prostatectomía radical (269), seguido de histerectomía (64) y plastia inguinal (33). Un total de 40 médicos certificados de cinco especialidades realizaron la totalidad de los procedimientos. Conclusiones: El iniciar un programa en un centro médico privado tiene diversas implicaciones. La creación de un comité de cirugía robótica integrado por médicos especialistas certificados en cirugía robótica de cada especialidad y autoridades del hospital para la acreditación de lineamientos tanto para la certificación como la recertificación de sus médicos puede beneficiar a programas como el nuestro por crear un centro de excelencia de cirugía robótica, disminuyendo complicaciones y mejorando resultados.

PALABRAS CLAVES: Da Vinci. Cirugía robótica. Experiencia inicial. Prostatectomía. Histerectomía.

\section{Robotic surgery at ABC Medical Center: first 500 procedures experience}

\section{Abstract}

Background. Robotic surgery is used in different surgical specialties worldwide. Objective. To documents the initial experience in a private hospital in the use robotic surgery in different surgical areas. Material and Methods. We included the first 500 robotic surgeries in our hospital in a 3 year period, documenting specialty and operative information. Results: Of the 500 patients, 367 (73.4\%) were male and 133 (26.4\%) female. The three most frequent surgeries performed were Radical Prostatectomy (269), Hysterectomy (64) an inguinal repair (33). A total of 40 certified surgeons ranging from 5 specialties performed the total number of surgeries. Conclusions. There are several implications in starting a robotic program in a private hospital setting. The creation of a robotic committee, formed by robotic certified physicians and hospital authorities, has helped in the certification process of its staff, lowering the complication rate and obtaining better surgical results.

KEY WORDS: Da Vinci. Robotic surgery. Initial experience. Prostatectomy. Hysterectomy. 


\section{Introducción}

La cirugía robótica se ha convertido en el abordaje convencional en un creciente número de procedimientos quirúrgicos. En el mundo los programas de cirugía robótica han crecido en los últimos años, estableciéndose como la cirugía de elección en gran número de centros y siendo adaptada por diversas especialidades quirúrgicas ${ }^{1-3}$. Desde la primera cirugía robótica realizada en marzo de 1997 por Cadière ${ }^{4}$, las indicaciones para esta plataforma quirúrgica han aumentado año a año. Aún hay procedimientos en los que puede existir cierta controversia con respecto a la ventaja que pudiera tener la cirugía robótica con respecto a la cirugía laparoscópica convencional ${ }^{5-7}$. Sin embargo, cada año aumenta el número de procedimientos en los que la cirugía robótica se establece como el abordaje de elección. Los avances y mejoras continuas en el equipo, así como el advenimiento de las nuevas plataformas robóticas en los próximos años tras el vencimiento de las patentes del equipo original, hará de la cirugía robótica el abordaje de elección en un mayor número de procedimientos quirúrgicos.

El inicio de cualquier programa de cirugía robótica puede ser abrumador por los retos que esto implica ${ }^{8,9}$. Tener personal médico, de enfermería, ingeniería biomédica y administrativo en sincronía para adaptar una nueva tecnología y asumir su papel en el nuevo proyecto puede resultar un reto complejo. Aunque la cirugía robótica puede tener grandes ventajas en cuanto a aprendizaje con respecto a la cirugía laparoscópica ${ }^{10}$, el crear un programa de cirugía robótica en cualquier institución médica implica tener cirujanos entrenados y en continuo entrenamiento, personal que aprenda el manejo de un equipo totalmente nuevo, con pasos quirúrgicos diferentes, y personal administrativo con miras de hacer crecer un programa diferente a lo que previamente habían manejado. El realizar esto en un hospital privado implica, además, el tener un modelo económico que esté dispuesto a crear un plan de negocios con el proveedor del equipo y las compañías aseguradoras.

Como reportamos en nuestra publicación anterior ${ }^{11}$, es crucial establecer un comité de cirugía robótica conformado por las diferentes especialidades involucradas para poder evaluar los casos de forma conjunta, autorizar procedimientos, y brindar un grupo médico que respalde con su experiencia en la cirugía robótica a los médicos nuevos en esta área y que justifique el uso de la cirugía robótica ante las compañías aseguradoras ${ }^{12-14}$. De esta forma, se ha podido minimizar complicaciones y establecer filtros que puedan garantizar el adecuado desarrollo de un programa nuevo con tantas implicaciones a nivel médico y administrativo ${ }^{15-17}$.

En esta publicación presentamos los primero 500 casos de cirugía robótica realizados en el Hospital $A B C$, así como los resultados obtenidos en esta parte de nuestra experiencia.

\section{Objetivo}

Evaluar los resultados de los primeros 500 casos de cirugía robótica en el Centro Médico ABC de enero del 2017 a diciembre del 2019, analizando el número de médicos certificados, las especialidades involucradas, tipos de cirugías, tiempos quirúrgicos y complicaciones. De igual manera, comparar los resultados entre los primeros 250 casos y los últimos 250 casos.

\section{Material y método}

El equipo robótico en el Hospital ABC Campus Observatorio es el Da Vinci S (Intuitive Surgical Inc., Sunnyvale, California). El equipo se instaló en diciembre del 2016 y la primera cirugía fue realizada el 23 de enero del 2017. Desde esa fecha hasta diciembre del 2019 se han realizado 500 cirugías robóticas.

Desde el inicio del programa, se creó un comité de cirugía robótica para la evaluación de los médicos certificados, credencialización y seguimiento de los casos de cirugía robótica con la finalidad de tener un control de los resultados y proyecciones del programa. El comité está integrado por médicos de las especialidades involucradas, ingenieros biomédicos, personal del cuerpo financiero y autoridades del hospital. Todos los casos fueron sometidos a consideración y aprobación por el comité de cirugía robótica previa programación. Analizamos la totalidad de cirugías realizadas en el hospital, así como especialidad, tipo de cirugía, número de cirujanos certificados, tiempos quirúrgicos, complicaciones, conversiones y días de estancia intrahospitalaria.

\section{Resultados}

En los primeros tres años del programa se han realizado 500 cirugías robóticas en el centro médico $\mathrm{ABC}$. De estas, 367 pacientes fueron de sexo masculino $(73.4 \%)$ y 133 de sexo femenino $(26.4 \%)$, con una 
edad promedio de 58 años (rango de 18 a 90 años). En la tabla 1 se muestra el desglose por rango de edad.

En la tabla 2 se muestra el desglose de cirugías por procedimiento. La cirugía más realizada fue la prostatectomía radical, con 269 cirugías, seguido de histerectomía, plastia inguinal y nefrectomía radical.

La cirugía urológica representó la mayoría de los procedimientos, contribuyendo con 346 cirugías (69.2\%), seguido de ginecología con 84 (16.8\%), cirugía general con $48(9.6 \%)$, cirugía de tórax con 18 (3.6\%) y cirugía oncológica con $4(0.8 \%)$.

El promedio de días de estancia intrahospitalaria fue de 2.95 días, con un rango de 1 a 28 días. La cistoprostatectomía asistida por robot fue uno de los procedimientos más complejos realizados, y representó el mayor número de días de estancia intrahospitalaria, con un promedio de 11 días (rango de 7 a 28). Excluyendo este procedimiento, el promedio de días de estancia intrahospitalaria baja a 2.6 días.

El tiempo quirúrgico promedio fue de 4 horas 35 minutos, con un rango de 45 minutos a 13 horas 40 minutos.

El sangrado promedio de todos los procedimientos fue de $250 \mathrm{ml}$, con un rango de 5-3,250 ml. De los 500 casos, 475 no requirieron transfusión, transfundiendo únicamente a 25 pacientes $(5 \%)$.

Al inicio del programa contábamos con seis médicos certificados con credenciales en el $A B C$ para realizar cirugía robótica. De estos, cuatro eran urólogos y dos eran ginecólogos. En el transcurso de los tres años del programa se han certificado un total de 34 médicos adicionales, teniendo en la actualidad 40 médicos certificados por Intuitive Surgical ${ }^{\circledR}$ y con autorización para realizar cirugía robótica en sus áreas de especialidad. El programa de entrenamiento para la certificación de los cirujanos siguió la normatividad mundial de la compañía. Consistió en 40 horas de entrenamiento en simulador, seguido de 10 horas de entrenamiento con modelos quirúrgicos, un curso certificado con simulador/modelo quirúrgico/modelo animal en uno de los centros certificados y finalmente la realización de una cirugía con el apoyo de un profesor certificado avalado por Intuitive Surgical ${ }^{\circledR}$ y la especialidad en cuestión.

El comité de cirugía robótica fungió como instancia calificadora para asegurar la correcta certificación o recertificación de los médicos, así como el poder estandarizar los insumos en cada uno de los procedimientos, mejorando de esta manera el factor económico.
Tabla 1. Distribución por rango de edad

\begin{tabular}{|c|c|c|}
\hline Rango de edad & Número & $\%$ \\
\hline $18-20$ & 5 & 1 \\
\hline $21-30$ & 11 & 2.2 \\
\hline $31-40$ & 40 & 8 \\
\hline $41-50$ & 70 & 14 \\
\hline $51-60$ & 119 & 23.8 \\
\hline $61-70$ & 173 & 34.6 \\
\hline $71-80$ & 80 & 16 \\
\hline $81-90$ & 2 & 0.4 \\
\hline
\end{tabular}

Tabla 2. Cirugías realizadas

\begin{tabular}{|c|c|}
\hline \multicolumn{2}{|c|}{$\begin{array}{l}\text { Primeros } 500 \text { casos } \\
\text { Periodo enero 2017- diciembre } 2019\end{array}$} \\
\hline Prostatectomía radical & 269 \\
\hline Histerectomía & 64 \\
\hline Plastia inguinal & 33 \\
\hline Nefrectomía radical & 25 \\
\hline Cistectomía & 21 \\
\hline Lobectomía pulmonar & 18 \\
\hline Miomectomía & 16 \\
\hline Nefrectomía parcial & 12 \\
\hline Funduplicatura & 8 \\
\hline Plastia ureteropiélica & 8 \\
\hline Reimplante ureteral & 5 \\
\hline Colecistectomía & 5 \\
\hline Linfadenectomía & 4 \\
\hline Ooforectomía & 4 \\
\hline Colposacrosuspensión & 2 \\
\hline Derivación biliodigestiva & 2 \\
\hline Tumor retroperitoneal & 2 \\
\hline Cirugía pancreática & 2 \\
\hline Total & 500 \\
\hline
\end{tabular}

Se presentaron 33 complicaciones en los primeros 500 casos (6.6\%), siendo la mayoría Clavien I. El sistema de clasificación de complicaciones Clavien-Dindo es un sistema uniformado, aceptado para el reporte de 
Tabla 3. Sistema de clasificación de Clavien-Dindo para clasificar las complicaciones quirúrgicas

\begin{tabular}{|c|c|}
\hline Grado & Definiciones \\
\hline । & $\begin{array}{l}\text { Cualquier desviación del curso postoperatorio normal } \\
\text { sin la necesidad de tratamiento farmacológico } \\
\text { o intervenciones quirúrgicas, endoscópicas y } \\
\text { radiológicas. Los regímenes terapéuticos aceptables } \\
\text { son los medicamentos como los antieméticos, } \\
\text { antipiréticos, analgésicos, diuréticos y electrolitos, y la } \\
\text { fisioterapia. Este grado también incluye las infecciones } \\
\text { de la herida abierta en la cabecera del paciente }\end{array}$ \\
\hline$\|$ & $\begin{array}{l}\text { Requiere tratamiento farmacológico con } \\
\text { medicamentos } \\
\text { distintos de los autorizados para las complicaciones } \\
\text { de grado I. También se incluyen las transfusiones de } \\
\text { sangre y la nutrición parenteral total }\end{array}$ \\
\hline III & $\begin{array}{l}\text { Requiere intervención quirúrgica, endoscópica o } \\
\text { radiológica }\end{array}$ \\
\hline IIla & Intervención que no se da bajo anestesia general \\
\hline IIIb & Intervención bajo anestesia general \\
\hline IV & $\begin{array}{l}\text { Complicación potencialmente mortal (incluidas } \\
\text { las complicaciones del sistema nervioso central: } \\
\text { hemorragia cerebral, infarto cerebral, hemorragia } \\
\text { subaracnoidea, pero con exclusión de los ataques } \\
\text { isquémicos transitorios) que requiere de la gestión de } \\
\text { la unidad de cuidados intermedios/intensivos }\end{array}$ \\
\hline IVa & Disfunción de un solo órgano (incluyendo la diálisis) \\
\hline $\mathrm{IVb}$ & Disfunción multiorgánica \\
\hline V & Muerte de un paciente \\
\hline Sufijo «d» & $\begin{array}{l}\text { Si el paciente padece una complicación en el } \\
\text { momento del alta se añade el sufijo «d» (de } \\
\text { discapacidad) al respectivo grado de complicación. } \\
\text { Esta etiqueta indica la necesidad de seguimiento para } \\
\text { evaluar la complicación al completo }\end{array}$ \\
\hline
\end{tabular}

complicaciones quirúrgicas ${ }^{18}$. Esta clasificación se muestra en la tabla 3. La distribución de complicaciones de acuerdo con la clasificación de Clavien-Dindo se muestra en la tabla 4. Las complicaciones más importantes fueron sangrado masivo (posterior a nefrectomía), perforación duodenal (posterior a cistoprostatectomía), sangrado transoperatorio que ameritó conversión, e infecciones que ameritaron reinternamiento.

\section{Discusión}

La cirugía robótica se ha convertido en el abordaje de elección en un creciente número de procedimientos quirúrgicos ${ }^{1,3,18,19}$. El advenimiento de nuevas tecnologías, como es el equipo Da Vinci Xi, así como lo anunciado por diversas compañías en cuanto a
Tabla 4. Complicaciones según la clasificación de Clavien

\begin{tabular}{|l|l|l|}
\hline Clavien I & 16 & $3.2 \%$ \\
\hline Clavien II & 6 & $1.2 \%$ \\
\hline Clavien IIIa & 5 & $1.0 \%$ \\
\hline Clavien IIIb & 6 & $1.2 \%$ \\
\hline
\end{tabular}

Tabla 5. Comparación entre los primeros 250 casos y los 500 casos

\begin{tabular}{|l|c|c|}
\hline Parámetro que comparar & 250 casos & 500 casos \\
\hline Días de ElH & 3.1 días & 2.95 días \\
\hline Tiempo quirúrgico & 4 h $50 \mathrm{~min}$ & 4 h $35 \mathrm{~min}$ \\
\hline Sangrado & $280 \mathrm{ml}$ & $250 \mathrm{ml}$ \\
\hline Complicaciones & $15(6 \%)$ & $33(6.6 \%)$ \\
\hline N. ${ }^{\circ}$ de cirujanos certificados & 26 & 40
\end{tabular}

EIH: estancia intrahospitalaria.

cambios y modificaciones en las nuevas plataformas, harán de la cirugía robótica un procedimiento económicamente más accesible y mejor adaptado para cada vez mayor número de procedimientos ${ }^{20,21}$. Es por ello que la creación de un programa de cirugía robótica, más que ser una novedad, es una necesidad actual para poder brindar a nuestra población tecnología de punta que es ya hoy en día un estándar quirúrgico bien establecido. La mejoría ergonómica y los resultados quirúrgicos están ya bien documentados en las diversas especialidades ${ }^{22-25}$. Iniciar un programa de cirugía robótica en nuestra institución ha requerido un cambio radical en muchas áreas, pero que al cabo de los años se ha llevado de forma satisfactoria. El inicio de cualquier programa nuevo debe ir de la mano con una baja morbilidad, bajos costos y adecuados resultados ${ }^{26}$, para poder así ofrecer al paciente un programa seguro y una adecuada transición de la cirugía abierta o laparoscópica convencional a esta nueva plataforma quirúrgica, y poder así cumplir con las expectativas de una nueva tecnología, de forma segura y sin complicaciones ${ }^{27,28}$.

En los últimos 250 casos se introdujo cirugía pancreática y un mayor número de procedimientos de cirugía torácica. El tener cirujanos que quieren explotar las capacidades de la plataforma permite que el programa de cirugía robótica crezca, ofreciendo procedimientos seguros y con buenos resultados en diferentes especialidades quirúrgicas. Mantener un nivel 
bajo de complicaciones depende de tener cirujanos experimentados que además estén conscientes de lo que implica el uso de un equipo quirúrgico nuevo. Las complicaciones se mantuvieron estables (6 y 6.6\%) en los primeros 250 casos y segundos 250 en estos tres años de experiencia. La cirugía robótica brinda la gran ventaja de tener dentro de su armamentario un simulador quirúrgico ${ }^{29}$ que ayuda tanto al entrenamiento de cirujanos nuevos como a reforzar las habilidades y el conocimiento de la consola de forma periódica. De esta manera se han establecido parámetros que permiten mantener a los cirujanos en constante entrenamiento, para poder así asegurar buenos resultados en sus intervenciones quirúrgicas con poca morbilidad.

El incremento en cirujanos robóticos en nuestra institución es un reflejo del interés en este abordaje quirúrgico, así como la necesidad de nuestra población de poder brindar cada vez mejores procedimientos. Es indispensable mantener estándares altos en cuanto a resultados y entrenamiento de los cirujanos para poder continuar con un programa quirúrgico exitoso ${ }^{30-32}$. En nuestra institución se incrementó de 26 a 40 cirujanos robóticos certificados entre los primero 250 y los segundos 250 casos como se muestra en la tabla $5^{33}$. Sin embargo, no hubo cambio estadístico significativo en complicaciones, sangrado, tiempo quirúrgico y tiempo de estancia intrahospitalaria entre estos dos periodos.

Los procedimientos urológicos siguen siendo los procedimientos que se realizan con mayor frecuencia. Sin embargo, la estadística mundial ha cambiado a favor de la cirugía ginecológica ${ }^{34-37}$.

\section{Conclusiones}

El programa de cirugía robótico en el Centro Médico $A B C$ ha demostrado ser efectivo desde el punto de vista de resultados, tiempos quirúrgicos y complicaciones, así como demostrar un avance tanto en el número de casos como en los resultados comparativos entre los primeros años y los años más recientes. El análisis de los casos nos permite evaluar la efectividad de programa y consolida el programa de cirugía robótica en nuestra institución como un programa sólido, con la posibilidad de involucrar más especialidades y mayor número de médicos.

\section{Conflicto de intereses}

Los autores declaran no tener conflicto de interés alguno.

\section{Financiamiento}

Los autores no recibieron patrocinio para llevar a cabo este artículo.

\section{Responsabilidades éticas}

Protección de personas y animales. Los autores declaran que para esta investigación no se han realizado experimentos en seres humanos ni en animales.

Confidencialidad de los datos. Los autores declaran que han seguido los protocolos de su centro de trabajo sobre la publicación de datos de pacientes.

Derecho a la privacidad y consentimiento informado. Los autores declaran que en este artículo no aparecen datos de pacientes.

\section{Bibliografía}

1. Schuessler WW, Schulam PG, Clayman RV, Kavoussi LR. Laparoscopic radical prostatectomy: initial short-term experience. Urology. 1997; 50(6):854-7

2. Brinkman W, de Angst I, Schreuder H, Schout B, Draaisma W, Verweij L, et al. Current training on the basics of robotic surgery in the Netherlands: Time for a multidisciplinary approach? Surg Endosc. 2017;31(1):281-7.

3. Farivar BS, Flannagan M, Leitman IM. General surgery residents' perception of robot-assisted procedures during surgical training. J Surg Educ. 2015;72(2):235-42.

4. Schuessler WW, Schulam PG, Clayman RV, Kavoussi LR. Laparoscopic radical prostatectomy: initial short-term experience. Urology. 1997;50:854-7.

5. Ahlering TE, Woo D, Eichel L, Lee DI, Edwards R, Skarecky DW. Robot-assisted versus open radical prostatectomy: a comparison of one surgeon's outcomes. Urology. 2004;63:819-22.

6. Hu JC, Gu X, Lipsitz SR, Barry MJ, D'Amico AV, Weinberg AC, et al. Comparative effectiveness of minimally invasive vs open radical prostatectomy. JAMA. 2009;302:1557-664.

7. Coelho RF, Rocco B, Patel MB, Orvieto MA, Chauhan S, Ficarra V, et al. Retropubic, laparoscopic, and robot-assisted radical prostatectomy: a critical review of outcomes reported by high-volume centers. J Endourol. 2010;24(12):2003-15.

8. Lumen N, van Praet C, de Troyer B, Fonteyne V, Oosterlinck W, Decaestecker K, et al. Safe introduction of robot-assisted radical prostatectomy after a training program in a high-volume robotic centre. Urol Int. 2013;91(2):145-52.

9. Moore BW, Dolat ME, McPartlin D, Mayer Grob B, Guruli G, Hampton LJ. Establishment of a new robotic prostatectomy program at a tertiary Veteran's Affairs medical center. J Robot Surg. 2013;7(2):171-5.

10. Gomes MTV, Costa Porto BTD, Parise Filho JP, Vasconcelos AL, Bottura BF, Marques RM. Safety model for the introduction of robotic surgery in gynecology. Rev Bras Ginecol Obstet. 2018;40(7):397-402.

11. Vásquez-Lastra C, Decanini TC, Maffuz-Aziz A, Alfaro AJ, Huante PJ. La cirugía robótica en el Centro Médico ABC: Informe de la formación del comité y la experiencia en los primeros 200 procedimientos realizados. Anales Médicos de la Asociación Médica del Centro Médico ABC. 2018;63(4):312-6

12. Chan F. Robotic-assisted surgical procedures are the future of gynaecology in Australasia. Aust N Z J Obstet Gynaecol. 2018;58(3):371-4.

13. Nelson B, Kaufman M, Broughton G, Cookson MS, Chang SS, Herrell SD, et al. Comparison of length of hospital stay between radical retropubic prostatectomy and robotic assisted laparoscopic prostatectomy. J Urol. 2007;177:929-31.

14. Zanagnolo V, Achilarre MT, Maruccio M, Garbi A. Might robotic-assisted surgery become commonplace in endometrial cancer treatment? Expert Rev Anticancer Ther. 2018;18(6):507-9.

15. Kosturakis AK, LaRusso KE, Carroll ND, Nicholl MB. First 100 consecutive robotic inguinal hernia repairs at a Veterans Affairs Hospital. J Robot Surg. 2018;12(4):699-704

16. Edelman DS. Robotic inguinal hernia repair. Am Surg. 2017;83(12):1418-21.

17. Arcerito M, Changchien E, Bernal O, Konkoly-Thege A, Moon J. Robotic inguinal hernia repair: Technique and early experience. Am Surg. 2016;82(10):1014-7. 
18. Mitropoulus D, Atribani W, Graefen M, Remzi M, Roupret M, Truss M. Reporting and grading of complications after urologic surgical proceduresf: an ad hoc EAU guidelines panel assessment and recommendations. Actas Urol Esp. 2013;37(1):1-11

19. Tsao AK, Smaldone MD, Averch TD, Jackman SV. Robot-assisted laparoscopic prostatectomy: the first 100 patients-improving patient safety and outcomes. J Endourol. 2009;23:481-4.

20. Kwon EO, Bautista TC, Blumberg JM, Jung H, Tamaddon K, Aboseif SR et al. Rapid implementation of a robot-assisted prostatectomy program in a large health maintenance organization setting. J Endourol. 2010; 24:461-5.

21. Box GN, Ahlering T. Robotic radical prostatectomy: long-term outcomes. Curr Opin Urol. 2008;18:173-9.

22. Yip SKh, Hong SG. Robotic radical prostatectomy in east Asia: development, surgical results, and challenges. Curr Opin Urol. 2010;20(1):80-5

23. Bolenz C, Gupta A, Hotze T, Ho R, Cadeddu JA, Roehrborn CG, et al Cost comparison of robotic, laparoscopic, and open radical prostatectomy for prostate cancer. Eur Urol. 2010;57:453-8.

24. Rocco B, Lorusso A, Coelho RF, Palmer KJ, Patel VR. Building a robotic program. Scand J Surg. 2009;98:72-5.

25. Steinberg PL, Merguerian PA, Bihrle W3rd, Seigne JD. The cost of learning robotic-assisted prostatectomy. Urology. 2008;72:1068-72.

26. Longo WE, Cheadle W, Fink A, Kozol R, DePalma R, Rege R, et al The role of the veterans affairs medical centers in patient care, surgical education, research and faculty development. Am J Surg 2005; 190:662-675

27. Link BA, Nelson R, Josephson DY, Lau C, Wilson TG. Training of urologic oncology fellows does not adversely impact outcomes of robot-assisted laparoscopic prostatectomy. J Endourol. 2009;23:301-5.

28. Tsao AK, Smaldone MD, Averch TD, Jackman SV. Robot-assisted laparoscopic prostatectomy: the first 100 patients-improving patient safety and outcomes. J Endourol. 2009;23(3):481-4
29. Laviana AA, Hu JC. A comparison of the robotic-assisted versus retropubic radical prostatectomy. Minerva Urol Nefrol. 2013;65(3):161-70.

30. Desai GS. Robotic surgery: is it right for India? J Robot Surg. 2018;12(4):725-6.

31. Lauterbach R, Matanes E, Lowenstein L. Review of robotic surgery in gynecology-The future is here. Rambam Maimonides Med J. 2017;8(2):e0019.

32. Truong M, Kim JH, Scheib S, Patzkowsky K. Advantages of robotics in benign gynecologic surgery. Curr Opin Obstet Gynecol. 2016;28(4):304-10.

33. Vásquez-Lastra C, Decanini TC, Maffuz-Aziz A, Alfaro AJ, Huante PJ. "La cirugía robótica en el Centro Médico ABC: Informe de la formación del comité y la experiencia en los primeros 200 procedimientos realizados". Anales Médicos de la Asociación Médica del Centro Médico ABC. 2018:63(4):312-6.

34. Heidenreich A, Bastian PJ, Bellmunt J, Bolla M, Joniau S, van der Kwast $\mathrm{T}$, et al. EAU guidelines on prostate cancer: Part 1-Screening, diagnosis, and treatment of clinically localised disease. Eur Urol. 2011:59:61-71.

35. Trinh QD, Sammon J, Sun M, Ravi P, Ghani KR, Bianchi M, et al. Perioperative outcomes of robot-assisted radical prostatectomy compared with open radical prostatectomy: results from the nationwide inpatient sample. Eur Urol. 2012;61(4):679-85.

36. Tewari A, Sooriakumaran P, Bloch DA, Seshadri-Kreaden U, Hebert AE, Wiklund $P$. Positive surgical margin and perioperative complication rates of primary surgical treatments for prostate cancer: A systematic review and meta-analysis comparing retropubic, laparoscopic, and robotic prostatectomy. Eur Urol. 2012;62:1-15.

37. Gandaglia G, Sammon JD, Chang SL, Choueiri TK, Hu JC, Karakiewicz PI, et al. Comparative effectiveness of robot-assisted and open radical prostatectomy in the postdissemination era. $\mathrm{J}$ Clin Oncol. 2014;32(14):216-21. 